\title{
Agradecimento aos avaliadores ad hoc de 2013
}

A ANPET e a Revista TRANSPORTES agradecem aos especialistas listados seguir pela colaboração prestada durante o ano de 2013. Sua experiência profissional e capacidade de julgamento foram fundamentais para julgar adequadamente a qualidade dos manuscritos recebidos. Sem a sua ajuda, teria sido impossível editar este periódico e produzir os números publicados em 2013.

Adalberto Leandro Faxina

Adelaida Pallavicini Fonseca

Adriana Leiras

Alessandro Vinícius Marques de Oliveira

Alexandre Gomes de Barros

Álvaro Seco

Ana Bastos

Ana Cristina Faria

Anderson Ribeiro Correia

André Bergsten Mendes

Antônio Galvão Novaes

Antônio Nélson Rodrigues da Silva

Áurea Silva de Holanda

Barbara Stolte Bezerra

Bruno Vieira Bertoncini

Carlos Alberto Faria

Carlos Felipe Grangeiro Loureiro

Carlos Henrique Rocha

Christine Tessele Nodari

Cira Souza Pitombo

Claudio Barbieri da Cunha

Claudio Jorge Pinto Alves

Claudio Toledo

Consuelo Alves da Frota

Daniel Anijar de Matos

Débora Pretti Ronconi

Ed Pinheiro Lima

Eduardo Camponogara

Enilson Medeiros dos Santos

Erika Cristine Kneib

Erinaldo Hilário Cavalcante

Flávio José Craveiro Cunto

Flávio Souza

Floriano C. M. Pires Jr.

Francisco Anuatti Neto

Francisco Gildemir Ferreira da Silva

Francisco Magalhães Gomes

Francisco Moraes Oliveira Neto

Francisco Thiago Sacramento Aragão

Geraldo Luciano de Oliveira Marques

Gislene Maia de Macêdo

Glauco Tulio Pessa Fabbri

Glaydston Mattos Ribeiro

Glicério Trichês

Heloisa Maria Barbosa

Homero Fernandes Oliveira

Hugo Tsugunobu Yoshida Yoshizaki

Ilton Curty Leal Jr.

Jamilla Emi Sudo Lutif Teixeira

João Alencar Oliveira Jr.

Joaquim José Guilherme de Aragão

Jorge Augusto Pereira Ceratti

Jorge Eduardo Leal Medeiros
José Aparecido Sorratini

José Reynaldo Setti

José Tadeu Balbo

Josiane Palma Lima

Kamilla Lima Vasconcelos

Lars Sanches

Laura Maria Goretti da Motta

Leise Kelli de Oliveira

Leni Figueiredo Mathias Leite

Lenise Grando Goldner

Leto Momm

Li Weigang

Licínio da Silva Portugal

Luciano Pivoto Specht

Luís Alberto H. do Nascimento

Luís Antonio Lindau

Manoel Mendonça de Castro Neto

Marcia Aps

Márcia Helena Macêdo

Marcio de Almeida D'Agosto

Marcone Jamilson Freitas Souza

Marcos Thadeu Queiroz Magalhães

Maria Alice Prudêncio Jacques

Maria Cristina Fogliatti de Sinay

Maria Leonor Alves Maia

Mário Ângelo Nunes de Azevedo Filho

Mateus Araújo e Silva

Michelle Andrade

Miriam Buss Gonçalves

Mônica Maria Mendes Luna

Nadja Glheuca da Silva Dutra

Nélio Domingues Pizzolato

Orivalde Soares da Silva Jr.

Pastor Willy Gonzales-Taco

Paulo CesarMarques da Silva

Renato Silva Lima

Rosângela dos Santos Motta

Rui A. Rodrigues Ramos

Sandra de Aguiar Soares

Sara Ferreira

Sergio Henrique Demarchi

Sergio Ronaldo Granemann

Suelly Helena de Araújo Barroso

Suely da Penha Sanches

Vânia Barcellos Gouvêa Campos

Verônica Teixeira Franco Castelo Branco

Vitória Pureza

Waldemiro de Aquino Pereira Neto

Washington Peres Nuñez

Werner Kraus Jr.

Yaeko Yamashita 